\title{
Der Europäische Pakt für psychische Gesundheit und Wohlbefinden: Kann die deutsche Psychiatrie vom Aus- tausch guter Praxisbeispiele auf EU-Ebene profitieren?
}

\author{
The European Pact for Mental Health and Well-Being: Can German Psychiatry \\ Benefit from the Exchange of Examples of Good Practice at the EU Level?
}

Autor

Institute
Anke Bramesfeld ${ }^{1,2}$

Medizinische Hochschule Hannover

European Commission, Directorate Health and Consumers, Luxembourg

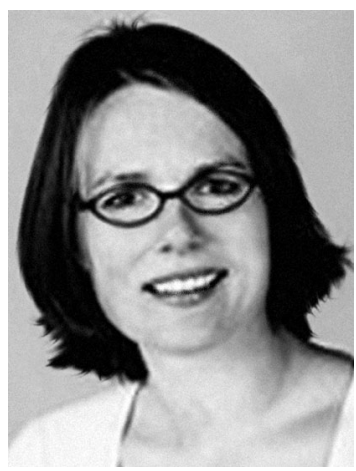

Dr. Anke Bramesfeld

\section{Impulse von der EU}

$\nabla$

In der auf psychische Gesundheit bezogenen Wissenschaftsszene in Deutschland ist die Europäische Union (EU) vor allem wegen ihrer Forschungsförderung bekannt. Tatsächlich kommen aber mehr Impulse für Gesundheitsversorgung und -forschung aus Europa als nur Forschungsförderung:

Im Juni 2008 rief die Europäische Kommission den Europäischen Pakt für psychische Gesundheit und Wohlbefinden ins Leben [1]. Dieser Pakt ist als ein Instrument der Kommission zu verstehen, über das die Sensibilisierung für die Bedeutung der psychischen Gesundheit für Europa gestärkt werden soll und eine strategische Ausrichtung der Aktivitäten in diesem Bereich erreicht werden soll.

\section{Ziele des Europäischen Paktes für psy- chische Gesundheit und Wohlbefinden $\nabla$}

Eine gute psychische Gesundheit der europäischen Bevölkerung und eine Verbesserung der Lebensbedingungen von Menschen mit psychischer Erkrankung liegen im strategischen Interesse der EU. Die strategischen Interessen der Europäischen Union finden sich in der Strategie von Lissabon für die Zeit bis 2010 und seit neuestenm in der Europa-Strategie 2020 [2]. Beide Strategien verknüpfen das Ziel einer starken und nachhaltigen Wirtschaft mit dem gesellschaftlichen Bedarf nach mehr Fokussierung auf Wissen, Information und Bildung sowie mit dem Lösen von sozialen Fragen, Bekämpfung von Armut und sozialer Ausgrenzung.

Vor diesem Hintergrund gewinnt die psychische Gesundheit der Bevölkerung eine neue Relevanz, denn eine auf Wissen und Information gegründete Gesellschaft bedarf der mentalen Kapazitäten und einer guten psychischen Belastbarkeit ihrer Mitglieder. Auch das Bekämpfen von sozialer Ausgrenzung sowie von sozialen und gesundheitlichen Ungleichheiten, ist eng mit Fragen der psychischen Gesundheit und den Möglichkeiten der gesellschaftlichen Teilhabe von Menschen mit psychischer Erkrankung und Behinderung verknüpft [3].

Für die Umsetzung der strategischen Ziele der Europäischen Union ist die Europäische Kommission als exekutives Organ zuständig. Maßnahmen im Bereich Gesundheit liegen jedoch primär in der autonomen Verantwortung der Mitgliedsstaaten. Direkte legislative Kompetenz besteht im Bereich Gesundheit auf europäischer Ebene nicht. Die Kommission kann im Bereich Gesundheit nur subsidiär wirken, Diskussionen anstoßen und mit Hilfe von Beispielen guter Praxis sowie Empfehlungen wirken.

Dies zu tun und mittels des Anstoßens von Diskussionen und Verbreiten von Beispielen guter Praxis, die Mitgliedstaaten und Akteure zu motivieren Maßnahmen zur Verbesserung der psychischen Gesundheit auf Bevölkerungsebene zu ergreifen, ist das Ziel des Europäischen Pakts für psychische Gesundheit und Wohlbefinden. Der Pakt möchte die Aktivitäten der Mitgliedstaaten im Bereich psychische Gesundheit bündeln und katalysieren. Hierfür konzentriert sich der Pakt auf 5 prioritäre Bereiche:

- Psychische Gesundheit von Kindern und Jugendlichen

- Prävention von Depression und Suizid

- Psychische Gesundheit im Alter

- Psychische Gesundheit am Arbeitsplatz

- Stigma und soziale Ausgrenzung

In diesen prioritären Bereichen werden thematische Konferenzen durchgeführt. Aufgabe der Konferenzen ist u.a. Aufmerksamkeit für die jeweiligen Themen herzustellen, Schlüsselaktivitä- 
ten zu identifizieren und gute Praktiken vorzustellen. In vielen Fällen kommen die guten Praktiken mit denen die Kommission arbeitet aus den wohlhabenden EU Staaten wie Großbritannien, Skandinavien, den Niederlanden, Spanien, Italien und auch Deutschland. Gerade Deutschland, das mit 80 Millionen Einwohnern der größte EU-Staat ist und dazu einer der wohlhabendsten, hat mit seinen vergleichsweise vielen akademischen Einrichtungen und einem elaborierten und sehr diversifizierten Gesundheitsversorgungssystem eine Reihe guter Praktiken vorzuweisen. Dies ist auch der Fall im Bereich der Förderung der psychischen Gesundheit und der Prävention psychischer Erkrankungen. Insgesamt wird zwar der Stand der Förderung der psychischen Gesundheit und Prävention psychischer Erkrankungen in Deutschland als eher schwach eingeschätzt [4], aber aufgrund der Größe des Landes und seiner Vielfalt finden sich lokal eine Reihe an guten Praktiken. Sie sind nur häufig nicht flächendeckend umgesetzt [5].

Welchen Gewinn kann Deutschland und insbesondere die deutsche Psychiatrie von einem Austausch von guten Praktiken auf EU-Ebene haben?

\section{Beispiele guter Praxis}

Deutschland kann vom Beispiel der anderen lernen. Es kann eigene vorhandene gute Ansätze wertschätzen und weiter verbessern. Beispiele guter Praxis in Deutschland im Bereich Prävention von Depression und Suizid sind z. B. die Bündnisse gegen Depression, die in Deutschland wie in keinem anderen EU-Land weite Verbreitung gefunden haben. Auch die Beschränkung der rezeptfreien Abgabemenge von Paracetamol auf $10 \mathrm{mg}$ als Maßnahme der Prävention von Suiziden im Affekt, ist als gute Praxis zu werten und erfolgt vor dem Hintergrund einer breiten Evidenzbasis. Lernen kann Deutschland aber an den folgenden Beispielen:

- Policy: Nationale Strategien, die Handlungsfelder definieren, aber darüber hinaus auch quantitative Erfolgsindikatoren benennen und diese messen, haben sich als hilfreich erwiesen um sowohl die Implementierung von Maßnahmen als auch die Forschung in diesem Bereich voranzutreiben. Ein gutes Beispiel hierfür findet sich in den Niederlanden, wo zurzeit eine nationale Depressionsstrategie umgesetzt wird. Diese umfasst sowohl Elemente der Prävention von Depression als auch die Implementierung von evidenzbasierten innovativen Versorgungsformen wie z. B. Stepped-Care-Ansätze [6, 7]. Für eine nationale Depressionsstrategie bestehen in Deutschland grundsätzlich gute Voraussetzungen: es existiert bereits ein nationales Gesundheitsziel Depression, das ein Grundinteresse und einen Konsens der Akteure begründet. Mit einem relativ gut ausgebauten Sekundärdatensystem durch die Abrechnungsdokumentationen der Kassenärztlichen Vereinigungen und der Krankenkassen sowie regelmäßigen Mikrozensuserhebungen verfügt Deutschland im Prinzip über Instrumente deren Nutzbarmachung für eine policybegleitende Dokumentation in Betracht gezogen werden kann.

- E-Health: Nutzen des Internets für Therapieangebote bei leichter und mittelschwerer Depression. Der Einsatz von eHealth-Interventionen bei der Behandlung von Depressionen ist in Deutschland bisher eher wenig entwickelt. Therapeutische Angebote aus dem Bereich der neuen Medien haben den Vorteil, dass der Zugang einfach ist, sie sind niederschwellig und haben das Potenzial kosteneffektiv zu sein [8]. Gute Bei- spiele für den Einsatz von E-Health bei der Behandlung von Depressionen finden sich u. a. in den Niederlanden [9, 10], wurden aber auch schon in Deutschland entwickelt.

- Kooperation von Allgemeinmedizin und psychiatrisch-psychotherapeutischer Versorgung: Dass eine engere Kooperation zwischen Allgemeinmedizin und spezialisierter psychiatrischpsychotherapeutischer Versorgung das Potenzial hat die Depressionsversorgung nachhaltig zu verbessern, ist inzwischen weitgehend akzeptiert [11,12]. Gute Beispiele hierfür finden sich z. B. in Norwegen, wo in verschiedenen Modellen versucht wird Psychotherapeuten aus den Einzelpraxen in Gemeinschaftskooperationen mit Allgemeinmedizinern zu bringen (s. http://ec.europa.eu/health/mental_health/docs/ ev_20091210_co31_en.pdf). Auch eine systematische Definition der klinischen „Pathways“, wie sie in Stepped-Care-Programmen vorgesehen sind, eignen sich die Zusammenarbeit zwischen Spezialisten und Generalisten zu erleichtern [7,1315].

- Suizidscreening: Neu in Gefängnisse eingewiesene Personen haben ein besonders großes Suizidrisiko. Ein einfaches SuizidScreening-Programm für diese Hochrisikogruppe wurde in Österreich entwickelt und umgesetzt. Es hat sich in der kontrollierten Anwendung als effektive Suizidpräventionsmaßnahme erwiesen [16].

Dies sind nur einige Beispiele guter Praxis aus dem Bereich Depression und Suizid. Mehr Anregungen finden Sie auf den Seiten der Europäischen Kommission im „EU-Kompass for Mental Health Action and Wellbeing“ [17]. Hier können die eigenen guten Praktiken mit denen anderer Mitgliedsstaaten verglichen werden und Anregungen geholt werden. Zudem verweisen Links sowohl zu Policy Papieren des Paktes, die zu jedem der 5 Prioritäten des Paktes Schlüsselaktionen identifizieren, als auch zu weiteren EU-Dokumenten, die für das Thema psychische Gesundheit relevant sind. Die Dokumente und Informationen des Kompasses stellen wichtige Werkzeuge dar, mit denen in der gesundheitspolitischen Diskussion Forderungen nach Verbesserungen im Bereich psychische Gesundheit in einen größeren Kontext gesetzt werden und nachdrücklicher gemacht werden können.

\section{Fazit}

$\nabla$

Zusammenfassend kann Deutschland von den europäischen Aktivitäten im Bereich psychische Gesundheit auf verschiedene Weise profitieren:

- Durch den Vergleich mit der guten Praxis in anderen EU-Mitgliedstaaten eigene Stärken und Schwächen identifizieren.

- Anregungen für Schwerpunkte im Bemühen um eine Verbesserung der psychischen Gesundheit sammeln.

- Durch die Weitergabe eigener guter Praktiken, diesen mehr Öffentlichkeit geben und aufwerten.

- Durch das Rekurrieren auf den europäischen Kontext eigenen Forderungen nach Verbesserungen mehr Nachdruck zu verleihen.

- Insbesondere die Deutsche Psychiatrie kann sich durch die europäischen Aktivitäten im Bereich Förderung der psychischen Gesundheit und Prävention psychischer Erkrankung anregen lassen, auch für die psychische Gesundheit relevante Sektoren wie Schulen, Arbeitsplätze oder Pflegeheime außerhalb des eigenen Versorgungssystems mehr Beachtung zu schenken. So kann ein Anschluss der klinischen Medizin an 
die vielen präventionsrelevanten Bereiche außerhalb des Gesundheitssektors hergestellt werden.

Ziel muss es sein, Strategien auf regionaler, nationaler und EUEbene zu entwickeln, wie die Förderung der psychischen Gesundheit und Prävention psychischer Erkrankungen insbesondere von Risikogruppen verbessert werden kann, wie der Zugang zu adäquater Versorgung verbessert und die gesellschaftliche Teilhabe psychisch kranker Menschen verstärkt werden kann. Aus deutscher Perspektive kann dabei der Blick nach Europa und in die anderen Mitgliedsstaaten Anregung und Unterstützung bieten [18].

\section{Literatur}

1 http://ec.europa.eu/health/mental_health/policy/index_en.htm 2 http://ec.europa.eu/eu2020/pdf/COMPLET\%20\%20DE\%20SG-201080021-06-00-DE-TRA-00.pdf

3 Riedel-Heller S, Richter D. Psychosocial interventions \& social inclusion - getting closer to those in need. Psychiat Prax 2008; 35: 213-215

4 Positionspapier GNMH http://gnmh.de/daten/20090207-positions papier.pdf

5 Riedel-Heller S, Bramesfeld A, Roick C et al. Call for more health services research. Psychiat Prax 2008; 35: 157-159

6 Smit F, Comijs H, Schoevers $R$ et al. Target groups for the prevention of late-life anxiety. Br J Psychiatry 2007; 190: 428-434

7 van't Veer-Tazelaar N, van Marwijk H, van Oppen P et al. Prevention of anxiety and depression in the age group of 75 years and over: a randomised controlled trial testing the feasibility and effectiveness of a generic stepped care programme among elderly community residents at high risk of developing anxiety and depression versus usual care. BMC Public Health 2006; 6: 186
8 Meyer B, Berger T, Caspar F et al. Effectiveness of a novel integrative online treatment for depression (Deprexis): randomized controlled trial. J Med Internet Res 2009; 11: e15

9 van Straten A, Cuijpers P, Smits N. Effectiveness of a web-based self-help intervention for symptoms of depression, anxiety, and stress: randomized controlled trial. J Med Internet Res 2008; 10: e7

10 Cuijpers $P$, van Straten A, Andersson G. Internet-administered cognitive behavior therapy for health problems: a systematic review. J Behav Med 2008; 31: 169-177

11 Bower P, Gilbody S, Richards D et al. Collaborative care for depression in primary care. Making sense of a complex intervention: systematic review and meta-regression. Brit J Psychiatry 2006; 189: 484-493

12 Gilbody S, Bower P, Fletcher J et al. Collaborative care for depression: a cumulative meta-analysis and review of longer-term outcomes. Arch Intern Med 2006; 166: 2304-2306

13 Sielk M, Altiner A, Janssen B et al. Prevalence and diagnosis of depression in primary care. A critical comparison between PHQ-9 and GPs' judgement. Psychiat Prax 2009; 36: 169-174

14 Bramesfeld A, Riedel-Heller S. Priorities in mental health services research. Psychiat Prax 2008; 35: 315-317

15 Glaesmer H, Gunzelmann T, Martin A et al. The impact of mental disorders on health care utilization and illness behaviour in the elderly. Psychiat Prax 2008; 35: 187-193

16 Frottier P, Koenig F, Seyringer $M$ et al. The distillation of „VISCI“: towards a better identification of suicidal inmates. Suicide Life Threat Behav 2009; 39: 376-385

17 http://ec.europa.eu/health/mental_health/eu_compass/index_en.htm

18 Riedel-Heller SG. Research in social psychiatry - addressing future challenges of health- and social systems. Neuropsychiatr 2009; 23: 249252 